\title{
Heavy precipitation episodes and cosmic rays variation
}

\author{
A. Mavrakis ${ }^{1}$ and S. Lykoudis ${ }^{2}$ \\ ${ }^{1}$ Department of Economic \& Regional Development, Panteion University, Athens, Greece \\ ${ }^{2}$ Institute of Environmental Research \& Sustainable Development, National Observatory of Athens, Greece
}

Received: 7 October 2005 - Revised: 18 January 2006 - Accepted: 23 January 2006 - Published: 20 February 2006

\begin{abstract}
In this paper an attempt is made to investigate the possible temporal correlation between heavy precipitation episodes and cosmic rays' activity, on various time scales. Cosmic rays measurements are sparse and cover less extended periods than those of precipitation. Precipitation is largely influenced by local climatic and even physiographic conditions, while cosmic rays' distribution is far more uniform over an area. Thus, in an effort to cover a larger range of climatic characteristics, each cosmic rays station was correlated with several nearby precipitation stations. Selected statistical methods were employed for the data processing. The analysis was preformed on annual, seasonal, monthly and daily basis whenever possible. Wet and dry regions and/or seasons seem to present a different response of precipitation to cosmic rays variations. Also Forbush decreases in most cases will not lead to heavy precipitation, yet this might be sensitive to precipitable water availability.
\end{abstract}

\section{Introduction}

Cosmic rays are energetic particles originating from both solar and non-solar sources. The latter are called galactic cosmic rays (GCR) and come mainly from outside the solar system. The number of GCRs that reach the surface of the Earth is influenced by solar activity, latitude, altitude, diurnal cycle, and Earth weather. Emissions of matter and electromagnetic fields from the Sun, namely solar wind, increase during high solar activity, making it harder for GCRs to penetrate the inner solar system and reach the Earth. Thus, the GCR intensity is lower when solar activity is high and vice-versa. The phenomenon presents an approximately 11 year periodicity (Manuel et al., 2002). Irregular decreases of GCR intensity at short time scales are of special interest. These anomalies are known as Forbush decreases (FDs), and are associated with magneto-hydrodynamic disturbances following solar coronal mass ejections (Pallé Bagó and Butler, 2000; Manuel et al., 2002).

Changes in local weather can also influence GCR fluxes. Strong atmospheric electric fields associated with thunderstorms have an effect on the propagation of GCR particles. Besides that, GCR intensity is dependent on altitude. The exact mechanism remains unknown, but increased cloudiness could be a plausible suggestion.

GCRs, on the other hand, may directly influence cloud formation through the production of cloud condensation and/or ice nuclei via GCR induced ionisation. Indirect mechanisms include modulation of the atmospheric electrical conductivity within the "global electric circuit" by GCR ionisation and subsequent effects on cloud microphysics through the process of electro-scavenging (Raspopov et al., 1998; Pallé et al., 2004). Furthermore, GCRs by triggering cloud formation might also be changing precipitation and variations in precipitation, potentially caused by changes in the GCR flux, thus having implications for the understanding of cloud and water vapour feedbacks.

Various possible effects of ionisation by either GCRs or solar activity itself on cloud formation and precipitation processes have been explored by several researchers. There is a long history of solar-climate studies, many of which have shown significant correlations between atmospheric parameters and solar variability (Marsh and Svensmark, 2000; Kniveton and Todd, 2001; Todd and Kniveton, 2001; Tsiropoula, 2003; Kniveton, 2004). Svensmark (1998), on the other hand, concludes that climate seems to be influenced by solar activity via the connection between GCRs and clouds, while heavy precipitation episodes, considered to be associated with GCRs variations, were found to result in greater radiative effects than solar output itself (Zhao et al., 2004). 


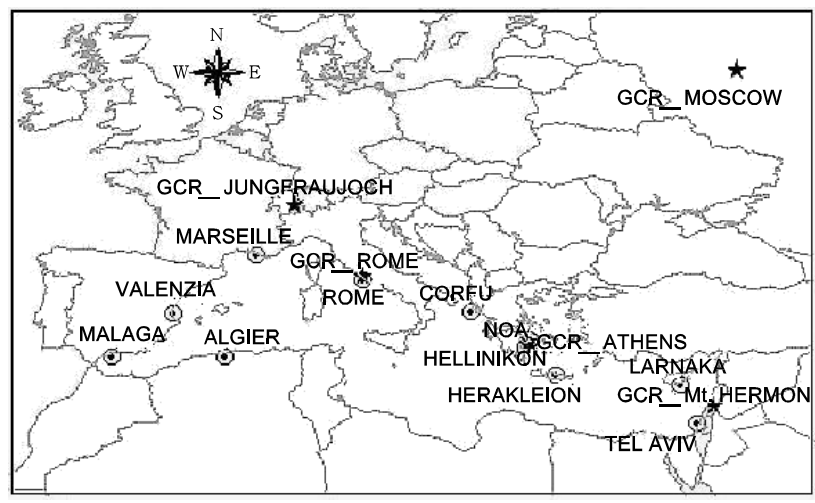

Fig. 1. Mediterranean GCR and precipitation stations.

Criticism (Jorgensen and Hansen, 2000; Sun and Bradley, 2002) and second thoughts on the relation between solar and GCRs variation and climate (Yu and Turco, 2001; Kniveton, 2004; Zhao et al., 2004), have focused on the lack of physical explanation on how very low energy fluxes, implicit in solar irradiance variations, are able to cause the observed climate variability that involves much higher energy fluxes. Even the long-term stability of the observed relationships has not been adequately explained yet. In this context there is a clear need for observational studies to assess the evidence for the operation of such mechanisms at climatic spatial and temporal scales.

The aim of this study is to examine the correlation between heavy precipitation episodes and GCR variations on an annual, seasonal and monthly basis, and to quantify the probability of a concurrency between FDs and heavy precipitation episodes, over the Mediterranean Region, considering several meteorological and GCR stations, across the area, for the period of 1958-2005.

\section{Data and methodology}

GCR data are recorded by ground-based neutron monitors measuring the low-energy part of the primary GCR spectrum. Although absolute GCR values present significant spatial variability, the normalized GCR variation, for different stations, remains quite similar (Svensmark and FriisChristensen, 1997). For the purpose of this study we obtained the GCRs datasets (10-20 GeV, pressure corrected) from all stations located around the Mediterranean and available online. The stations used are Rome, Jungfraujoch, Mt Hermon, and Athens plus Izmiran Moscow that served as a second reference - the first being Rome - due to its longterm uniform dataset. Daily precipitation data were obtained from the European Climate Assessment archive (Klein Tank et al., 2002), and from the National Observatory of Athens (NOA). Stations were selected so as to cover the whole extent of the Mediterranean (Fig. 1) while, at the same time, having data series covering a period matching the GCR dataset from Izmiran and Rome (1958-2005).
The precipitation stations can be sorted into two groups: one between the 30th and 40th parallel (Malaga, Valenzia, Algier - Dar El Beida, Athens - NOA and Hellinikon, Herakleion, Larnaka and Tel Aviv) and a second between the 40th and 50th parallels (Marseille, Rome - Ciampino and marginally Corfu), thus following the zonal GCR distribution.

The relative variation of the GCRs counted at each station was examined using normalized counts. Typical normalization by subtracting the long-term mean and dividing by the corresponding standard deviation was used in order to avoid the problem of different scales between the stations. Possible relationships between heavy precipitation episodes and GCR variation were investigated using monthly frequencies of precipitation episodes exceeding $10 \mathrm{~mm}$ and $20 \mathrm{~mm}$ calculated from daily data. Normalized episode frequencies were correlated to the respective normalized GCR time series from Rome, the Mediterranean station with the longest dataset.

In order to investigate whether it is an FD that triggers a heavy precipitation episode or the other way around, we calculated the probability of having a heavy precipitation episode before or after an FD. Three precipitation thresholds were used, namely 10,20 and $40 \mathrm{~mm}$, and seven time lags ranging from -3 to +3 days from the FD incident. A GCR count decrease was considered to be an FD when the difference between the daily count and the 45-day running mean exceeded a certain threshold. A decrease threshold of 3.5\% provided comparable numbers of FD events for Izmiran and Rome - about 350 for the whole period- and was also appropriate for Athens.

Finally, for each time lag, two-way contingency tables were created giving the probability at which an FD would coincide with a precipitation episode. Izmiran GCR was paired against all stations, since its daily data set was considered to be more uniform than that of Rome. Also, Athens GCR data were paired against the two precipitation stations of Athens.

\section{Results}

Normalized GCR counts from Izmiran and Rome are almost identical throughout the examined period (1958-2005) with a Pearson correlation coefficient 0.95 , thus supporting the possibility of, alternatively, using either data set. All other stations present similar variation patterns even though with different degrees of variability. Mt. Hermon and Athens are much more variable than the rest of the stations during the most recent years (2000-2005) (Fig. 2).

Furthermore, Pearson correlation coefficients between normalized precipitation episode frequencies $(\mathrm{RR}>10 \mathrm{~mm}$ and $\mathrm{RR}>20 \mathrm{~mm}$ ) for each precipitation station and normalized GCR counts for Rome were calculated using annual, seasonal (winter-summer) and monthly data for the period 1958-2005. The coefficients are listed in Table 1 and selected graphs are presented in Fig. 3. It is evident that the response of heavy precipitation incidences to the GCR variations across the examined area is far from being uniform. 
Table 1. Pearson correlation coefficients between normalized precipitation episodes and normalized GCR counts for Rome for the period 1958-2005.

\begin{tabular}{lrrrrrrrrr}
\hline & \multicolumn{2}{c}{ Annual } & \multicolumn{2}{c}{ Summer } & \multicolumn{2}{c}{ Winter } & \multicolumn{2}{c}{ Monthly } & Annual precipitation \\
\hline Station & $\mathrm{RR}>10$ & $\mathrm{RR}>20$ & $\mathrm{RR}>10$ & $\mathrm{RR}>20$ & $\mathrm{RR}>10$ & $\mathrm{RR}>20$ & $\mathrm{RR}>10$ & $\mathrm{RR}>20$ & \\
Malaga & 0.031 & 0.064 & -0.001 & -0.034 & 0.013 & -0.095 & 0.053 & 0.061 & 430 \\
Valenzia & -0.195 & -0.342 & 0.157 & 0.168 & -0.180 & 0.249 & -0.009 & -0.057 & 430 \\
Algier & 0.155 & 0.229 & -0.057 & -0.03 & 0.123 & 0.044 & 0.078 & 0.083 & 641 \\
Marseille & 0.200 & 0.228 & 0.161 & 0.164 & 0.273 & 0.233 & 0.111 & 0.094 & 575 \\
Rome & 0.060 & 0.009 & 0.025 & 0.010 & 0.037 & 0.135 & -0.006 & 0.003 & 758 \\
Corfu & 0.034 & 0.058 & 0.169 & 0.101 & 0.098 & 0.122 & 0.057 & 0.059 & 376 \\
NOA & 0.136 & 0.093 & -0.064 & 0.065 & 0.193 & 0.061 & 0.057 & 0.033 & 371 \\
Hellinikon & 0.256 & 0.145 & -0.011 & 0.152 & 0.291 & 0.017 & 0.104 & 0.062 & 472 \\
Herakleion & 0.155 & 0.146 & -0.078 & -0.206 & 0.093 & -0.199 & 0.081 & 0.075 & 319 \\
Larnaka & -0.040 & -0.141 & -0.144 & -0.047 & -0.011 & 0.106 & 0.027 & -0.008 & 469 \\
TelAviv & -0.054 & -0.100 & 0.183 & 0.053 & -0.057 & -0.087 & 0.053 & 0.022 & \\
\hline
\end{tabular}

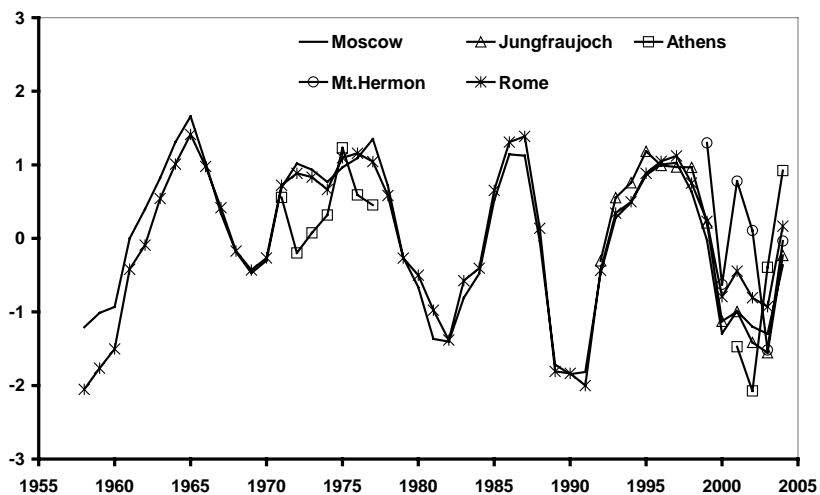

Fig. 2. Annual normalized GCR data for all stations.

The correlation between precipitation episodes and GCR varies both spatially and in terms of the examined time scale, in accordance to previous findings (Trimble et al., 1997). Annual data present higher correlations, ranging from $+25 \%$ to $-34 \%)$ compared to seasonal ( $+27 \%$ to $-20 \%)$ or monthly $(+11 \%$ to $-6 \%)$. This decline in correlation coefficients with increasing temporal resolution is due to the fact that precipitation has a marked interannual variation which is not present in GCR flux. Drier stations tend to present negative correlations and the same applies to summer periods. Under moderate to low precipitable water availability, GCR activity reduction leads to reduced condensation nuclei availability, thus to less competition between those and raindrops. This allows the later to grow and precipitate. On the other hand, under abundant precipitable water conditions the condensation nuclei produced by the GCR do not hinder raindrop growth, eventually becoming raindrops themselves.

Since FDs are of special interest we investigated the probability of having a precipitation episode within the period zero to three days after an FD incident. A high probability would suggest that FDs cause or at least favor precipitation episodes. On the other hand, we have also investigated

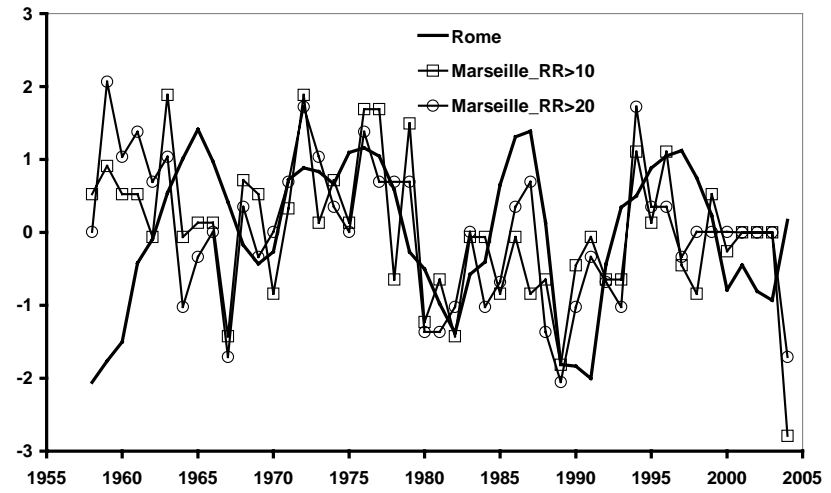

Fig. 3a. Normalized annual GCR counts from Rome and normalized precipitation episode frequency $(\mathrm{RR}>10-$ square and $\mathrm{RR}>20$ - circle) in Marseille

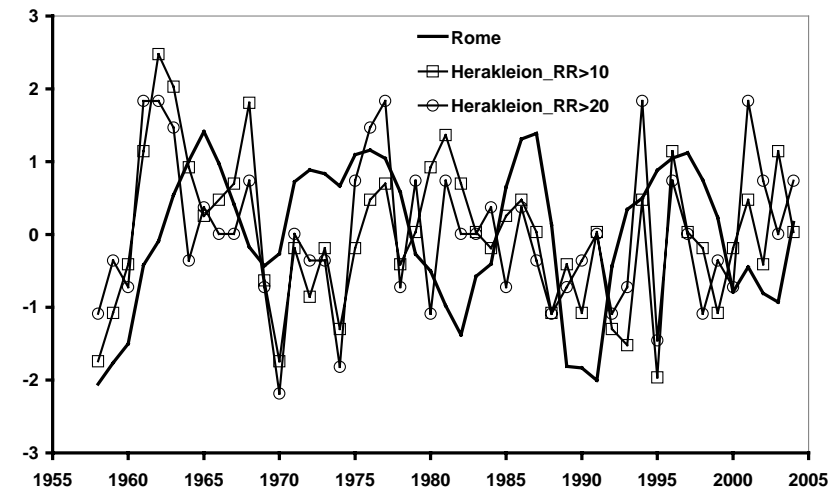

Fig. 3b. Normalized annual GCR counts from Rome and normalized precipitation episode frequency $(R R>10$ - square and $R R>20$ - circle) in Herakleion.

the probability of having an episode up to three days before the FD. Such an instance would indicate that the FD was the result of the precipitation episode even though this could only apply to data from neighboring GCR and precipitation 


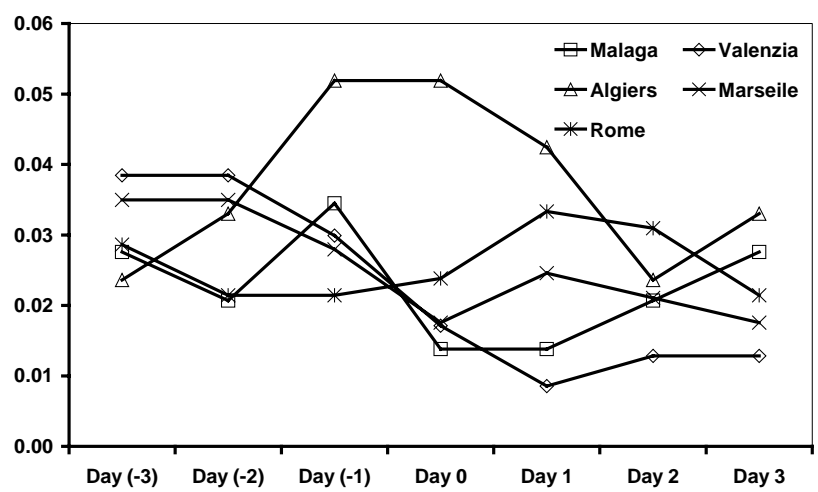

Fig. 4a. Coincidence probabilities between Izmiran FDs and summer mild episodes (RR>10 mm) for Western Mediterranean.

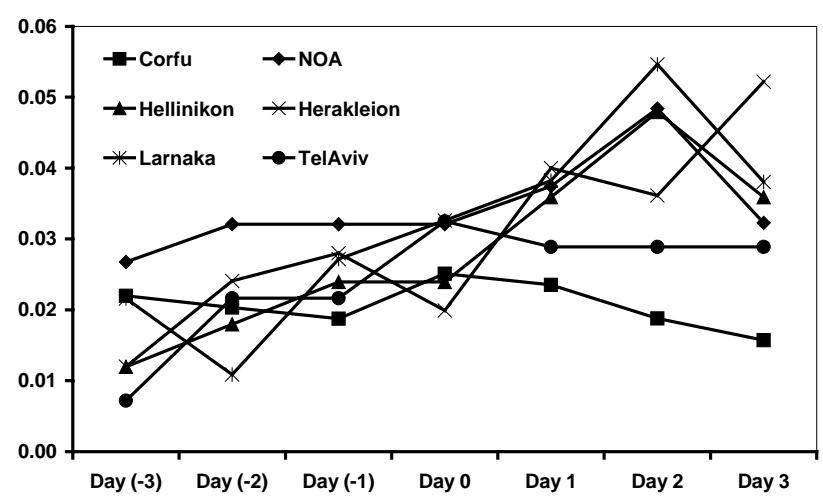

Fig. 4b. Coincidence probabilities between Izmiran FDs and winter episodes ( $R R>20 \mathrm{~mm}$ ) for Eastern Mediterranean.

stations, due to the local character of precipitation episodes. Winter probabilities are quite different from that of summer, both in terms of distribution shape and magnitude. In several cases some of the distributions are practically non-existent. As expected, annual probabilities lay somewhere in between of the seasonal ones, so only the latter will be discussed herein. Overall the observed probabilities, when using the Izmiran GCR station, are rather small - up to 9\%, rendering any remarks indicative only.

For mild episodes ( $R R>10)$, during summer, most of the Eastern Mediterranean stations present higher probabilities for a precipitation episode 1 day after an FD as compared to the days 0 and -1 in accordance to other works (Kniveton, 2004). Western Mediterranean stations present the opposite behavior (Fig. 4). More intense episodes ( $R R>20$ ), during winter, are more frequent 1 and 2 days after the FD for the drier Eastern stations (NOA, Hellinikon, Tel Aviv) while the rest of the Eastern Mediterranean stations present higher probabilities during the FD day (Day 0). Western stations again have a different, almost invariant, behavior (Fig. 4). Severe episodes $(\mathrm{R}>40 \mathrm{~mm})$ and FDs interaction is even less clear due to the relative scarceness of such episodes.

The examination of precipitation episode and FD coincidence on a local scale also provides some interesting re-

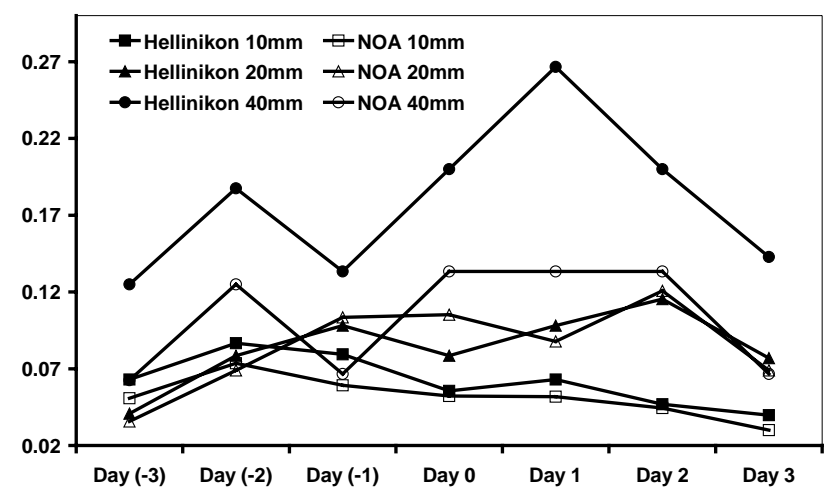

Fig. 5. Winter coincidence probabilities between Athens FDs and precipitation episodes in NOA and Hellinikon.

sults. Figure 5 illustrates that the probability of an episode occurrence 1-2 days after an FD increases along with the considered episode threshold. Also, a possible effect of local weather on the GCRs count could only be suggested for the mild episodes $(\mathrm{RR}>10 \mathrm{~mm})$. This, however, might be a misreading of the figure, since one could argue that the figure actually shows a drop in probability after the FD day rather than an increase before. This, of course, would imply the existence of a background probability of coincidence. This explanation is consistent with the previous remark according to which when there is limited precipitable water the competition between ionization-produced condensation nuclei and raindrops limits the probability of a precipitation episode, or its intensity. On the contrary when there is enough precipitable water, as is often the case in winter, the aforementioned competition has no effect, thus allowing the ionization-produced condensation nuclei to grow and produce significant, heavy precipitation episodes.

\section{Conclusions}

In this paper an attempt was made to obtain some observational inferences on the possible relationship between the variation of GCR flux, depending mainly on magnetic latitude, and precipitation. Instead of considering all precipitation episodes, this work focused on heavy precipitation episodes that are more sensitive to factors, like condensation nuclei abundance, possibly related to GCR flux variations.

The analysis presented above supports a distinction between wet and dry regions and/or seasons regarding the response of precipitation to GCR variations. Condensation nuclei and their competition with raindrops over the available moisture in the air could be the key to this differentiation, yet supporting this inference would require a far more detailed analysis than the one attempted herein.

Finally, even though it seems that an FD in most cases will not lead to a precipitation episode, one should consider that if we could distinguish those cases with an adequate amount of available precipitable water the results might have been different. 
Acknowledgements. The authors would like to thank the Cosmic Ray Groups of: Nuclear and Particle Physics Section, Physics Department, University of Athens, Greece; Cosmic Ray Department of Solar-Terrestrial Division of Izmiran-Moscow, Russia; Universita degli Studi "Roma Tre", Dipartimento di Fisica "Edoardo Amaldi", Italy; Physikalisches Institut, University of Bern, Switzerland; Israel Space Weather and Cosmic Ray Centre \& "Emilio Segre" Israel-Italy Observatory; for providing the GCR data. Also special thanks are due to the European Climate Assessment Group at KNMI, Netherlands for providing the precipitation data.

Edited by: V. Kotroni and K. Lagouvardos

Reviewed by: anonymous referee

\section{References}

Jorgensen, T. S. and Hansen, A. W.: Comments on "Variation of cosmic ray flux and global cloud coverage - a missing link in solar-climate relationships" by Henrik Svensmark and Eigil Friis-Christensen (J. Atmos. Solar-Terr. Phys., 59, 1225-1232, 1997), J. Atmos. Solar-Terr. Phys., 62, 73-77, 2000.

Klein Tank, A. M. G., Wijngaard, J. B., Konnen, G. P., Bohm, R., Demaree, G., Gocheva, A., Mileta, M., Pashiardis, S., Hejkrlik, L., Kern-Hansen, C., Heino, R., Bessemoulin, P., MullerWestermeier, G., Tzanakou, M., Szalai, S., Palsdottir, T., Fitzgerald, D., Rubin, S., Capaldo, M., Maugeri, M., Leitass, A., Bukantis, A., Aberfeld, R., Van Engelen, A. F. V., Forland, E., Mietus, M., Coelho, F., Mares, C., Razuvaev, V., Nieplova, E., Cegnar, T., Lopez, J. A., Dahlstrom, B., Moberg, A., Kirchhofer, W., Ceylan, A., Pachaliuk, O., Alexander, L. V., and Petrovic, P.: Daily dataset of 20th-century surface air temperature and precipitation series for the European Climate Assessment, Int. J. Climatol., 22, 1441-1453, data and metadata available at: http://eca.knmi.nl, 2002.

Kniveton, D. R. and Todd, M. V.: On the relationship of cosmic rays flux and precipitation, Geophys. Res. Lett., 28, 1527-1530, 2001.

Kniveton, D. R.: Precipitation, cloud cover and Forbush decreases in galactic cosmic rays, J. Atmos. Solar-Terr. Phys., 66, 11351142, 2004.
Manuel, K. O., Ninham, W. B., and Friberg, E. S.: Super fluidity in the solar interior: Implications for solar eruptions and climate, J. Fusion Energy, 21, 3-4, 2002.

Marsh, D. N. and Svensmark, H.: Low cloud properties influenced by cosmic rays, Phys. Rev. Lett., 85, 5004-5007, 2000.

Pallé Bagó, E. and Butler, C. J.: The influence of cosmic rays on terrestrial clouds and global warming, Astron. Geophys., 41, 4, 18-22, 2000.

Pallé, E., Butler, C. J., and Brien, K. O.: The possible connection between ionization in the atmosphere by cosmic rays and low level clouds, J. Atmos. Solar-Terr. Phys., 66, 1779-1790, 2004.

Raspopov, O.M., Shumilov, O.I., Kasatkina, E.A.: Cosmic rays as the main factor of influence of solar variability on climatic and atmospheric parameters. Biophysics, 43, 5, 858 - 863, 1998.

Sun, B. and Bradley, R.: Solar influences on cosmic rays and cloud formation: A reassessment, J. Geophys. Res., 107(D14), 42114223, 2002.

Svensmark, H. and Friis-Christensen, E.: Variation of cosmic ray flux and global cloud coverage - A missing link in solar-climate relationship, J. Atmos. Solar-Terr. Phys., 59, 1225-1232, 1997.

Svensmark, H.: Influence of cosmic rays on Earths climate, Phys. Rev. Lett., 81, 5027-5030, 1998.

Todd, M. C. and Kniveton, D. R.: Changes in cloud cover associated with Forbush decreases of galactic cosmic rays, J. Geophys. Res., 106(D23), 32 031-32 041, 2001.

Trimble, P. J., Santee, E. R., and Neidrau, C. J.: Including the effects of solar activity for more efficient water management: An application of neural networks, Second International Workshop on Artificial Intelligence Applications in Solar-Terrestrial Physics, Sweden, July 1997.

Tsiropoula, G.: Signatures of solar activity in meteorological parameters, J. Atmos. and Solar-Terr. Phys., 65, 469-482, 2003.

Yu, F. and Turco, R. P.: Ultrafine aerosol formation via ionmediated nucleation, Geophys. Res. Lett., 27, 883-887, 2000.

Zhao, J., Han Y.-B., and Li, Z.-A.: The effect of solar activity on the annual precipitation in the Beijing area, Chin. J. Astron. Astrophys., 4, 2, 189-197, 2004. 